\title{
Evolução da mortalidade infantil por causas evitáveis no estado do Piauí: 2008 a 2017
}

\author{
Evolution of child mortality from preventable causes in the state of Piauí: 2008 to 2017 \\ Evolución de la mortalidad infantil por causas prevenibles en el estado de Piauí: 2008 a \\ 2017
}

Rafael Everton Assunção Ribeiro da Costa ${ }^{1 *}$, Carlos Eduardo Moura Carvalho Rocha ${ }^{1}$, Luciana Tolstenko Nogueira ${ }^{1}$, Lucielma Salmito Soares Pinto ${ }^{1}$, Julio Cesar Saraiva Santos ${ }^{1}$, Eduardo Salmito Soares Pinto ${ }^{2}$.

\section{RESUMO}

Objetivo: Avaliar a evolução da mortalidade infantil por causas evitáveis no estado do Piauí entre 2008 e 2017. Métodos: Estudo ecológico e retrospectivo, a partir de dados secundários do Departamento de Informática do Sistema Único de Saúde e do Instituto Brasileiro de Geografia Estatística referentes ao Piauí, entre 2008 e 2017. Calcularam-se os coeficientes de Pearson referentes à correlação entre os óbitos infantis evitáveis e as porcentagens de municípios com IDHM muito baixo e baixo e de indivíduos com cadastro no Programa de Saúde da Família (PSF), com cálculo da significância estatística destas correlações. Resultados: De 2008 a 2017, registraram-se 8.088 óbitos infantis no Piauí: 5.688 evitáveis (70,33\%), 2.114 não-evitáveis $(26,14 \%)$ e 286 por causas mal definidas $(3,54 \%)$. Prevaleceram óbitos de crianças do sexo masculino, etnia negra ou parda e no período neonatal (0-27 dias). Houve correlação entre o perfil socioeconômico (IDHM) e de saúde (cobertura do PSF) e o percentual de óbitos infantis evitáveis neste estudo $(r=+0,573$ e -0,961, $p=0,0306$ e $<0,0001$, respectivamente). Conclusão: Apesar da tendência de queda, a mortalidade infantil total e por causas evitáveis no Piauí ainda foi elevada entre 2008 e 2017.

Palavras-chave: Mortalidade infantil, Correlação de dados, Epidemiologia.

\begin{abstract}
Objective: To evaluate the evolution of infant mortality due to preventable causes in the state of Piauí between 2008 and 2017. Methods: Retrospective and ecological study, using secondary data from the Database of the Brazilian Unified Health System and the Brazilian Institute of Geography and Statistics referring to the Piauí, between 2008 and 2017. Pearson's coefficients for the correlation between preventable infant deaths and the percentages of municipalities with very low and low MHDI and individuals registered in the Family Health Program (PSF) were calculated, with calculation of these correlations's statistical significance. Results: From 2008 to 2017, 8,088 infant deaths were recorded in Piauí: 5,688 preventable (70.33\%), 2,114 non-preventable (26.14\%) and 286 from ill-defined causes (3.54\%). Deaths of male children, black or mixed race and in the neonatal period (0-27 days) prevailed. There was a correlation between the socioeconomic profile (MHDI) and health (coverage of the PSF) and the percentage of preventable infant deaths in this study $(r=+0.573$ and $0.961, p=0.0306$ and $<0.0001$, respectively). Conclusion: Despite the downward trend, total infant mortality and preventable causes in Piauí was still high between 2008 and 2017.
\end{abstract}

Keywords: Infant mortality, Correlation of data, Epidemiology.

\section{RESUMEN}

Objetivo: Evaluar la evolución de la mortalidad infantil por causas prevenibles en el Piauí entre 2008 y 2017. Métodos: Estudio retrospectivo y ecológico, utilizando datos secundarios de la base de datos del Sistema Único de Salud y del Instituto Brasileño de Geografía y Estadística refiriéndose al Piauí, entre 2008 y 2017. Se calcularon los coeficientes de Pearson para la correlación entre las muertes infantiles prevenibles y los porcentajes de municipios con IDHM muy bajo y bajo y las personas registradas en el Programa de Salud de

${ }^{1}$ Universidade Estadual do Piauí (UESPI), Teresina - PI. *E-mail: rafassuncao.rafael@gmail.com

${ }^{2}$ Centro Universitário de Saúde, Ciências Humanas e Tecnológicas do Piauí (UNINOVAFAPI), Teresina -PI. 
la Familia (PSF), con el cálculo de significación estadística. Resultados: De 2008 a 2017, se registraron 8.088 muertes infantiles en Piauí: 5.688 prevenibles (70,33\%), 2.114 no prevenibles $(26,14 \%)$ y 286 por causas mal definidas $(3,54 \%)$. Prevalecieron las muertes de niños varones, de raza negra o mestiza y en el período neonatal (0-27 días). Hubo una correlación entre el perfil socioeconómico (IDHM) y la salud (cobertura del PSF) y el porcentaje de muertes infantiles prevenibles en este estudio $(r=+0.573$ y $-0.961, p=0.0306$ y $<0.0001$, respectivamente). Conclusión: A pesar de la tendencia a la baja, la mortalidad infantil total y por causas prevenibles en Piauí aún fueron altas entre 2008 y 2017.

Palabras clave: Mortalidad infantil, Correlación de datos, Epidemiología.

\section{INTRODUÇÃO}

Apesar de, naturalmente, ser difícil mensurar o bem-estar social por meio de um indicador por conta da complexidade envolvida, há alguns indicadores que se constituem como chaves na avaliação da qualidade de vida de uma população (SZWARCWALD CL, et al., 1997). Um destes indicadores é o coeficiente de mortalidade infantil, por estar relacionado a uma ampla gama de parâmetros socioeconômicos e de saúde, servindo, portanto, como um importante termômetro do desenvolvimento de uma sociedade (SZWARCWALD CL, et al., 1997).

A despeito das dificuldades técnicas em registrar e mensurar o real número de óbitos infantis e de nascimentos ano a ano, os métodos atuais de estimativa da mortalidade infantil conseguiram se consolidar como bons indicadores situacionais deste fenômeno a nível local, regional e nacional, sendo também importantes para mensurar a qualidade de vida populacional e direcionar políticas públicas em saúde (SZWARCWALD CL, et al., 1997; RIPSA, 2008; SARDINHA LMV, 2014; CARVALHO RAS, et al., 2015; PACHECO CP, 2010). Tecnicamente, o coeficiente de mortalidade infantil é avaliado por uma fórmula, cujo numerador consiste em óbitos de menores de um ano e o denominador no número de nascidos vivos, que, para critérios de análise, é estudado de acordo com a população, usualmente para cada 100.000 nascidos vivos, e com períodos de tempo determinados (RIPSA, 2008; SARDINHA LMV, 2014; CARVALHO RAS, et al., 2015; PACHECO CP, 2010).

A literatura classifica a mortalidade infantil em grupos etários, a partir do número de dias vividos, quais sejam: neonatal precoce ( 0 a 6 dias), neonatal tardia ( 7 a 27 dias) e pós-neonatal ( 28 a 364 dias), com o objetivo de discriminar melhor a causa mortis, pois óbitos no período neonatal são mais relacionados à qualidade dos serviços de saúde prestados a uma determinada população, enquanto aqueles no período pósneonatal possuem maior relação com o ambiente em que as crianças estão inseridas (RIPSA, 2008; SARDINHA LMV, 2014; CARVALHO RAS, et al., 2015; PACHECO CP, 2010).

Atualmente, o Sistema Único de Saúde (SUS) divide as causas de óbitos infantis em categorias relacionadas à evitabilidade por imunização, atenção à mulher durante a gestação e o parto, atenção ao recém-nascido, diagnóstico e tratamento adequados e promoção e atenção em saúde; além destas, figuram também discriminadas as causas consideradas não-evitáveis e mal-definidas (BRASIL, 2019).

O uso dessas categorias com base no CID-10 e de outras variáveis relacionadas aos óbitos infantis por causas evitáveis, não evitáveis e mal definidas, como os dados epidemiológicos grupo etário, sexo, etnia e região geográfica, pelo Departamento de Informática do Sistema Único de Saúde (DATASUS) fornece dados acerca da mortalidade infantil, que são de muito valor para a gestão de sistemas de saúde e estudos epidemiológicos (SARDINHA LMV, 2014; BRASIL, 2019).

É notável que a mortalidade infantil está bastante relacionada à qualidade de vida de uma população. No Brasil, a maioria das mortes infantis é evitável, ou seja, decorre de má assistência à saúde e/ou de condições ambientais e socioeconômicas precárias e poderia ser menos frequente, em um melhor contexto (NASCIMENTO SG, et al., 2014; RUOFF AB, et al., 2018; SANDERS LSC, et al., 2017).

Assim sendo, levando em conta a relevância desse marcador, este estudo se propõe a analisar a evolução da mortalidade infantil por causas evitáveis no estado do Piauí e correlacioná-la com o perfil socioeconômico (IDHM) e de saúde (cobertura do PSF) no período compreendido entre 2008 e 2017. 


\section{MÉTODOS}

\section{Local do estudo}

Segundo dados do Instituto Brasileiro de Geografia Estatística (IBGE), o estado do Piauí apresenta uma extensão territorial de $251.756,515 \mathrm{Km}^{2}$, sendo dividida em 4 macrorregiões: Semiárido, Meio-norte, Litoral e Cerrados e uma população estimada em 3.281.480 pessoas para o ano de 2020 (IBGE, 2020). Referente aos parâmetros socioeconômicos, o Piauí apresenta um Índice de Desenvolvimento Humano (IDH) de 0,646, o qual se configura como o quarto menor do país (BRASIL, 2019; IBGE, 2010).

\section{Desenho do estudo}

Trata-se de um estudo ecológico, retrospectivo, a partir de dados secundários coletados dos sites do Departamento de Informática do Sistema Único de Saúde (DATASUS) [www.datasus.gov.br] e do Instituto Brasileiro de Geografia Estatística (IBGE) [www.ibge.gov.br]. A amostra foi composta por todas as crianças nascidas vivas, bem como aquelas que foram a óbito antes de 1 ano de vida no estado do Piauí entre 2008 e 2017.

\section{Coleta dos dados}

Os dados foram extraídos do Sistema de Informações sobre Mortalidade (SIM), Sistema de Informações sobre Nascidos Vivos (SINASC) e Sistema de Informação de Atenção Básica (SIAB) do DATASUS, de acordo com os seguintes critérios: óbitos ou nascimentos de acordo com a residência da mãe e segundo macrorregiões como nível geográfico. As variáveis coletadas foram: grupos etários (neonatal precoce, neonatal tardio e pós-neonatal), grupos de causas (evitáveis, mal definidas e não-evitáveis), sexo e etnia.

No tocante ao grupo evitáveis, os dados foram agrupados dentro de cada categoria com base no CID-10 específico de óbitos infantis evitáveis (reduzíveis por ações de imunização, reduzíveis por adequada atenção à mulher na gestação, reduzíveis por adequada atenção à mulher no parto, reduzíveis por adequada atenção ao recém-nascido, reduzíveis por ações de diagnóstico e tratamento adequado e reduzíveis por ações de promoção da saúde vinculadas a ações de atenção em saúde). Os casos em que as variáveis consideradas para a coleta de dados do SIM foram ignoradas durante o registro não foram incluídos isoladamente, mas expostos no número total de casos de cada variável.

Também foram coletados os Índices de Desenvolvimento Humano Municipal (IDHM) de todos os 224 municípios do Piauí, de acordo com as macrorregiões, segundo o Censo 2010 do IBGE, bem como o número de indivíduos cadastrados no Programa de Saúde da Família (PSF) em 2010, de acordo com o SIAB, para realização dos estudos de correlação entre os óbitos e os indicadores de perfil socioeconômico e de saúde, respectivamente, e a mortalidade infantil por causas evitáveis em cada macrorregião por meio do coeficiente de Pearson.

\section{Análise Estatística}

Utiliza-se o coeficiente de correlação de Pearson quando se deseja associar linearmente duas variáveis. Seus valores variam entre -1 e +1 , sendo que um coeficiente igual a -1 indica uma relação inversa perfeita, igual a 0 indica que não há correlação linear e igual a +1 , uma relação direta perfeita (FILHO DBF e JÚNIOR JAS, 2009; COSTA REAR, et al., 2020). A tabulação dos dados ocorreu a partir do programa TabNet Win32 3.0. Em seguida, os dados foram exportados para o Microsoft $\circledast$ Office Excel for MAC 2011, programa em que foi feita a análise. Calcularam-se os coeficientes referentes à correlação entre os óbitos infantis evitáveis e as porcentagens de municípios com IDHM baixo e muito baixo e de indivíduos com cadastro no Programa de Saúde da Família (PSF). À posteriori, realizou-se o teste $t$ de Student para amostras independentes na avaliação da significância estatística destas correlações. Valores de $p<0,05$ foram considerados significantes.

\section{Aspectos Éticos}

Neste estudo, utilizaram-se apenas dados secundários presentes em bases de dados de domínio público, os quais não contém a identificação dos sujeitos. Portanto, não houve a necessidade de aprovação por um Comitê de Ética em Pesquisa (CEP). Porém, os preceitos contidos na Resolução do Conselho Nacional de Saúde n. 466/12 foram observados. 


\section{RESULTADOS}

De 2008 a 2017, registraram-se no SINASC 490.338 nascimentos no estado do Piauí e, no SIM, 8.088 óbitos infantis: 5.688 evitáveis (70,33\%), 2.114 não-evitáveis $(26,14 \%)$ e 286 por causas mal definidas (3,54\%). Com relação ao momento do óbito: $72,48 \%$ aconteceram no neonatal e $27,49 \%$ no pós-neonatal. No tocante às variáveis sexo e etnia, o sexo masculino foi o mais afetado em todos os anos da série histórica, representando cerca de $54,9 \%$ do total de mortes infantis de 2008 a 2017, bem como a etnia negra ou parda, correspondendo a, aproximadamente, $70,7 \%$ do total de óbitos.

O Coeficiente de Mortalidade Infantil (CMI) total no Piauí foi de 18,4/1000 nascidos vivos em 2008 e de 15,6/1000 nascidos vivos em 2017, o que evidencia uma redução de, aproximadamente, 15,2\%. O componente neonatal (0-27 dias) foi o responsável por esse declínio, apresentando uma redução de $23,1 \%$ entre 2008 e 2017, enquanto o pós-neonatal (28-365 dias) apresentou incremento de 6,30\% nesse período.

O CMI por causas evitáveis, por sua vez, foi de 13,5/1000 nascidos vivos em 2008 e de 10,3/1000 nascidos vivos em 2017 (redução de 23,7\%); enquanto o CMI por causas não evitáveis (4,2/1000 nascidos vivos em 2008 e 5,0/1000 nascidos vivos em 2017) apresentou aumento percentual de 19,0\% no mesmo período, e o por causas mal-definidas (0,68/1000 nascidos vivos em 2008 e 0,33/1000 nascidos vivos em 2017) mostrou queda de $51,5 \%$ entre 2008 e 2017 (Figuras 1 e 2).

Figura 1- Distribuição de coeficientes de mortalidade infantil no estado do Piauí de 2008 a 2017 por grupo etário.

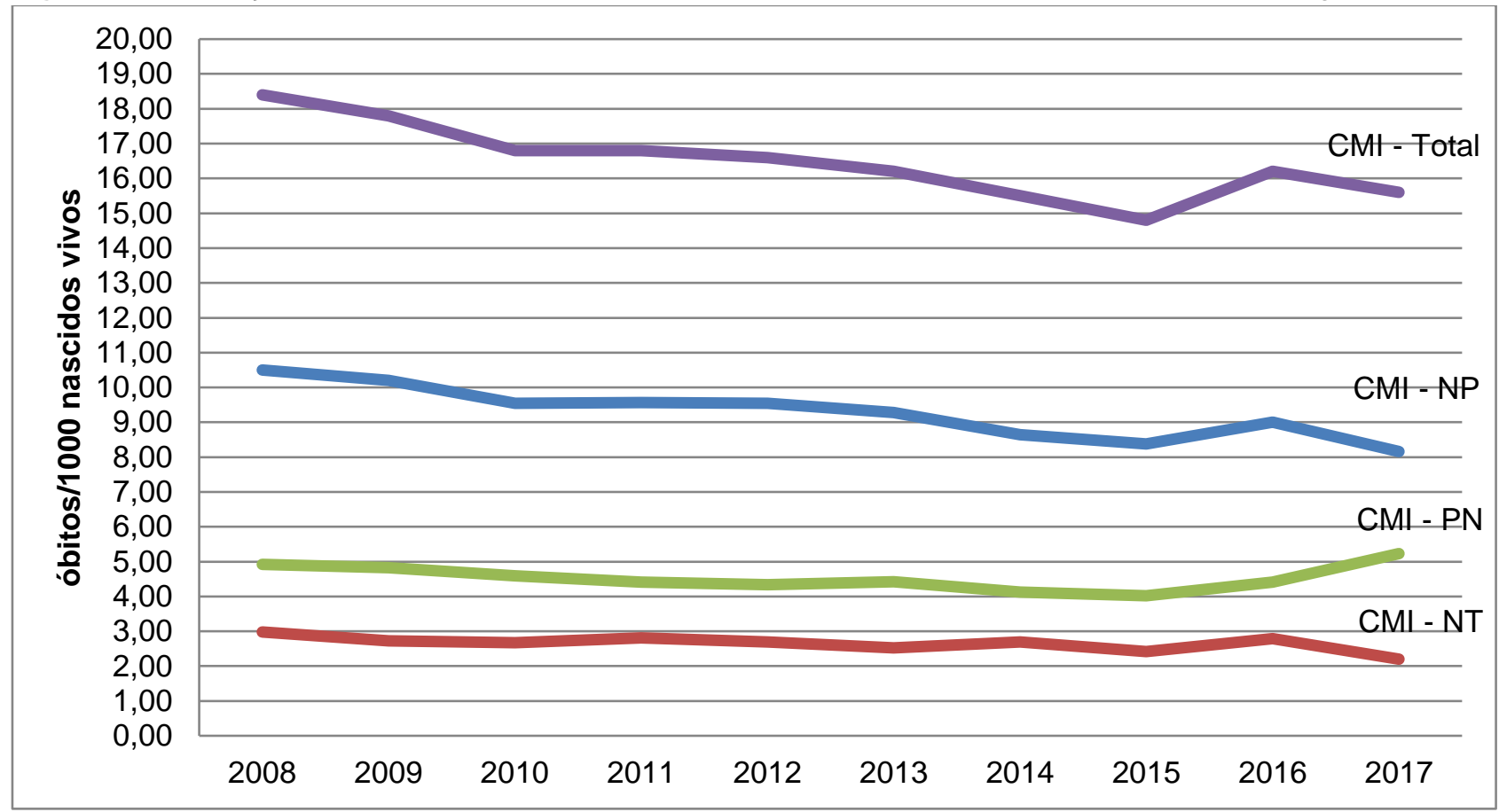

Legenda: CMI - NP: Coeficiente de Mortalidade Infantil neonatal precoce; CMI - NT: Coeficiente de Mortalidade Infantil neonatal tardio; CMI - PN: Coeficiente de Mortalidade Infantil pós-neonatal; CMI - Total: Coeficiente de Mortalidade Infantil total.

Fonte: Costa REAR, et al., 2019; dados extraídos do Sistema de Informações sobre Mortalidade (SIM) e Sistema de Informações sobre Nascidos Vivos (SINASC).

Analisando-se as categorias de causas evitáveis com base no CID-10 de óbitos infantis no Piauí entre 2008 e 2017, a categoria reduzíveis pela atenção à mulher na gestação foi a que apresentou maior número de óbitos infantis evitáveis em todos os anos entre 2008 e 2017, representando cerca de 46,4\% do total de óbitos infantis por causas evitáveis no Piauí de 2008 a 2017, e a categoria reduzíveis pelas ações de imunização foi a que apresentou o menor número de óbitos infantis, com apenas 2 casos no período (Figura 3). 
Figura 2 - Distribuição de coeficientes de mortalidade infantil no estado do Piauí de 2008 a 2017 por grupo de causa mortis.

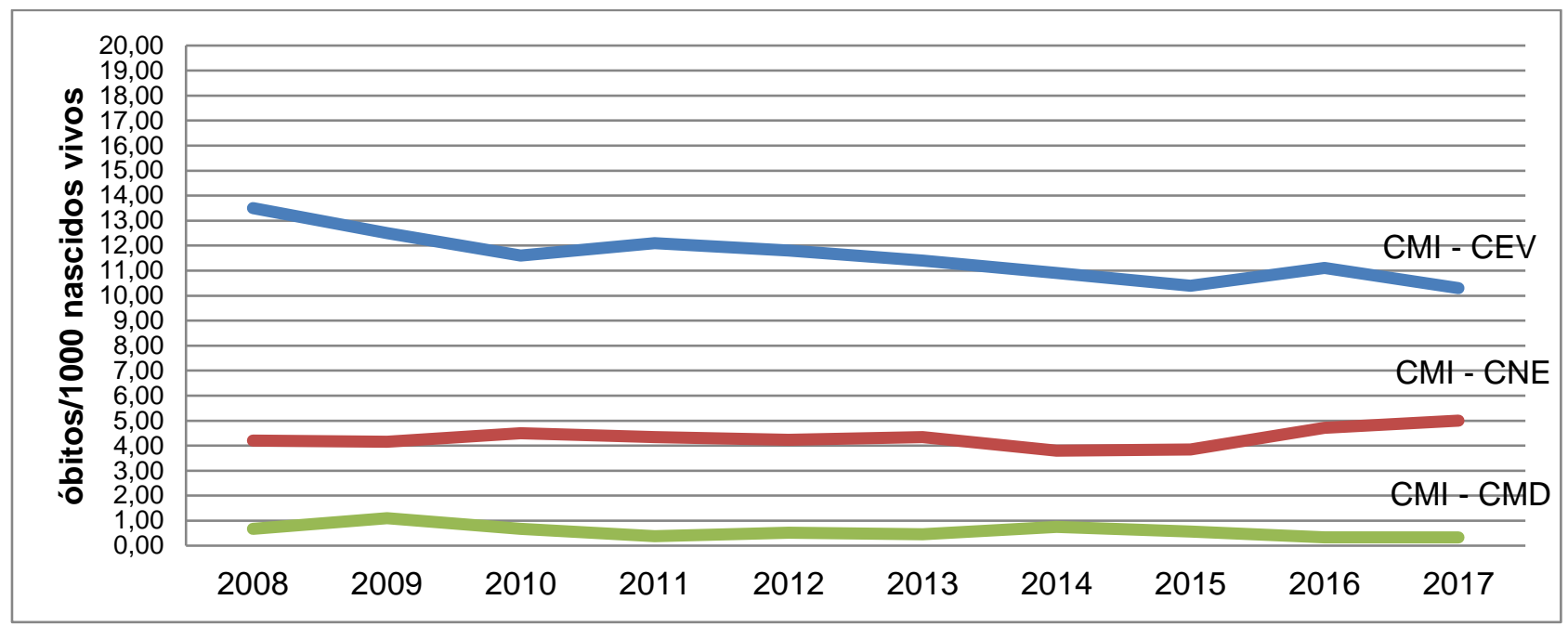

Legenda: CMI - CEV: Coeficiente de Mortalidade Infantil por causas evitáveis; CMI - CNE: Coeficiente de Mortalidade Infantil por causas não-evitáveis; CMI - CMD: Coeficiente de Mortalidade Infantil por Causas maldefinidas.

Fonte: Costa REAR, et al., 2019; dados extraídos do Sistema de Informações sobre Mortalidade (SIM) e Sistema de Informações sobre Nascidos Vivos (SINASC).

Figura 3 - Óbitos infantis evitáveis por categorias com base no CID-10 no estado do Piauí entre 2008 e 2017.

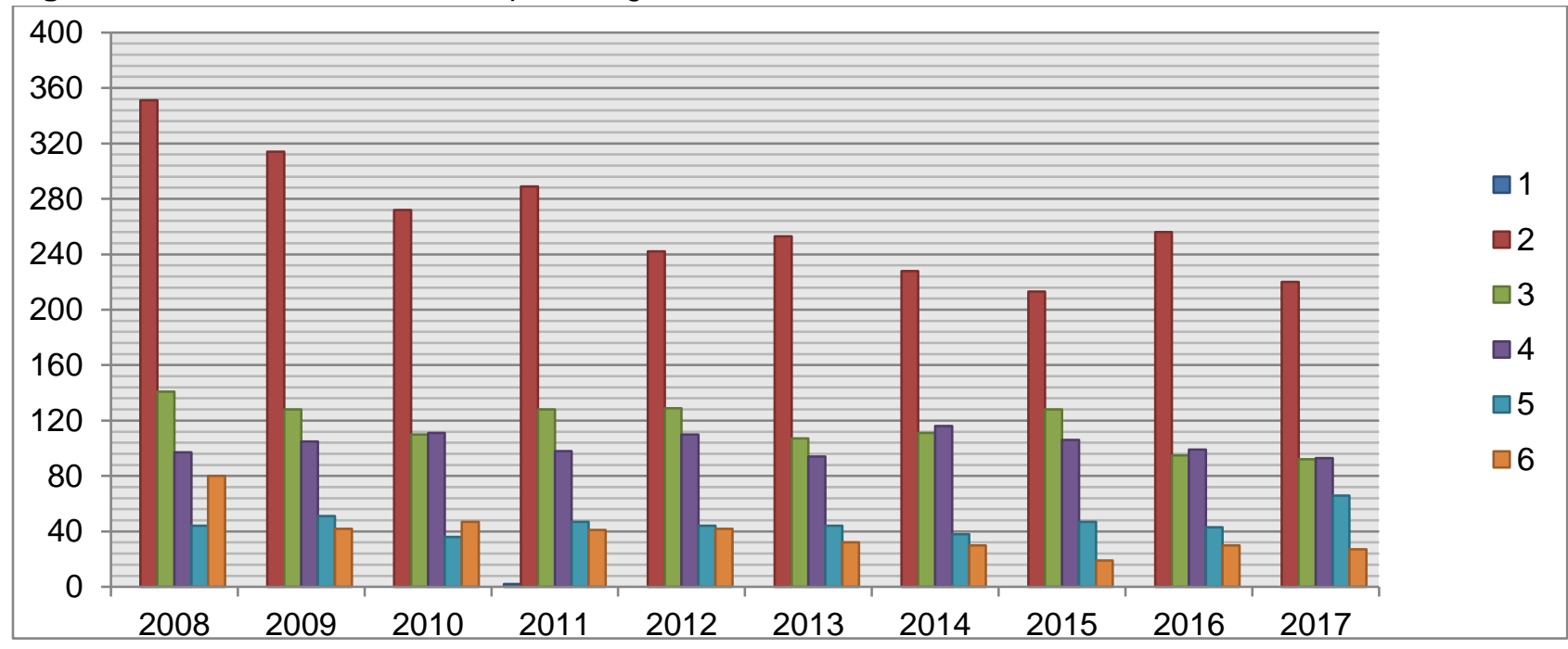

Legenda: 1: Reduzíveis por ações de imunização; 2: Reduzíveis por adequada atenção à mulher na gestação; 3: Reduzíveis por adequada atenção à mulher no parto; 4: Reduzíveis por adequada atenção ao recém-nascido; 5: Reduzíveis por ações de diagnóstico e tratamento adequado; 6: Reduzíveis por ações de promoção da saúde vinculadas a ações de atenção em saúde.

Fonte: Costa REAR, et al., 2019; dados extraídos do Sistema de Informações sobre Mortalidade (SIM).

Com relação às macrorregiões, a maior mortalidade foi observada na região Meio-Norte, com 3.396 óbitos no período (41,99\%), seguida por 1.739 óbitos, na Litoral (21,50\%), 1.488, na Cerrados $(18,40 \%)$ e 1.465 óbitos infantis na Semiárido (18,11\%) (Tabelas 1 e 2). O percentual de óbitos evitáveis no período foi de $72,6 \%$ na macrorregião Litoral, 71,5\%, na Semiárido, 69,4\%, na Meio-norte, e 68,7\% na Cerrados. Com relação aos indicadores socioeconômicos e de saúde, segundo o Censo 2010 do IBGE, a porcentagem de munícipios com IDHM baixo ou muito baixo, foi de $90,9 \%$, na macrorregião Litoral, $83,8 \%$, na Cerrados, $80,0 \%$, na macrorregião Semiárido e 72,3\%, na Meio-norte. De acordo com dados de 2010 do DATASUS, observou- 
se que $96,5 \%$ dos indivíduos da macrorregião Cerrados possuíam cadastro no Programa de Saúde da Família (PSF), seguido por, 95,2\%, da Meio-norte, 94,4\%, da macrorregião Semiárido e 93,5\%, da Litoral. Percebeuse relação direta entre os percentuais de óbitos evitáveis por macrorregião e IDHM baixo ou muito baixo $(r=$ $+0,573, p=0,0306)$; e relação inversa entre os percentuais de óbito e de cadastro no PSF $(r=-0,961, p<$ 0,0001 ) (Tabelas 1 e 2).

Tabela 1. Óbitos infantis por grupos etários nas macrorregiões do estado do Piauí entre 2008 e 2017.

\begin{tabular}{|c|c|c|c|c|c|c|c|c|c|c|c|c|}
\hline \multirow{2}{*}{$\begin{array}{l}\mathbf{M} \\
\mathbf{R} \\
\end{array}$} & Ano & 2008 & 2009 & 2010 & 2011 & 2012 & 2013 & 2014 & 2015 & 2016 & 2017 & Total \\
\hline & \multicolumn{12}{|l|}{ Grupo etário } \\
\hline \multirow{5}{*}{$\begin{array}{l}\mathbf{S} \\
\mathbf{A}\end{array}$} & NP & 99 & 111 & 91 & 92 & 110 & 72 & 74 & 79 & 78 & 60 & 866 \\
\hline & NT & 20 & 25 & 23 & 26 & 19 & 14 & 21 & 16 & 20 & 12 & 196 \\
\hline & $\mathrm{PN}$ & 46 & 54 & 38 & 42 & 41 & 36 & 29 & 41 & 32 & 43 & 402 \\
\hline & Total & 166 & 190 & 152 & 160 & 170 & 122 & 124 & 136 & 130 & 115 & 1465 \\
\hline & NP & 219 & 201 & 179 & 194 & 167 & 178 & 175 & 166 & 161 & 186 & 1826 \\
\hline \multirow{3}{*}{$\begin{array}{l}\mathbf{M} \\
\mathbf{N}\end{array}$} & NT & 81 & 61 & 65 & 67 & 65 & 64 & 54 & 63 & 58 & 54 & 632 \\
\hline & PN & 105 & 92 & 90 & 95 & 86 & 87 & 91 & 84 & 91 & 116 & 937 \\
\hline & Total & 405 & 355 & 334 & 356 & 318 & 329 & 320 & 313 & 310 & 356 & 3396 \\
\hline \multirow{5}{*}{ LI } & $\mathrm{NP}$ & 128 & 131 & 117 & 114 & 97 & 97 & 86 & 106 & 85 & 69 & 1030 \\
\hline & NT & 35 & 25 & 19 & 24 & 28 & 22 & 26 & 28 & 30 & 20 & 257 \\
\hline & $\mathrm{PN}$ & 56 & 47 & 47 & 33 & 42 & 51 & 45 & 42 & 38 & 51 & 452 \\
\hline & Total & 219 & 203 & 183 & 171 & 167 & 170 & 157 & 176 & 153 & 140 & 1739 \\
\hline & NP & 107 & 78 & 85 & 80 & 84 & 84 & 79 & 62 & 99 & 81 & 839 \\
\hline C & NT & 21 & 28 & 25 & 24 & 17 & 17 & 28 & 12 & 23 & 21 & 216 \\
\hline \multirow[t]{2}{*}{$\mathbf{E}$} & PN & 52 & 53 & 52 & 51 & 39 & 31 & 33 & 31 & 46 & 44 & 432 \\
\hline & Total & 180 & 160 & 162 & 155 & 140 & 132 & 140 & 105 & 168 & 146 & 1488 \\
\hline
\end{tabular}

Legenda: MR: Macrorregiões; SA: Semiárido; MN: Meio-norte; LI: Litoral; CE: Cerrados; NP: Neonatal precoce; NT: Neonatal tardio; PN: Pós-neonatal.

Fonte: Costa REAR, et al., 2019; dados extraídos do Sistema de Informações sobre Mortalidade (SIM).

Tabela 2 - Óbitos infantis por grupos de causas nas macrorregiões do estado do Piauí entre 2008 e 2017.

\begin{tabular}{|c|c|c|c|c|c|c|c|c|c|c|c|c|}
\hline \multirow[b]{2}{*}{ MR } & Ano & 2008 & 2009 & 2010 & 2011 & 2012 & 2013 & 2014 & 2015 & 2016 & 2017 & Total \\
\hline & \multicolumn{12}{|l|}{$\begin{array}{l}\text { Grupo de } \\
\text { causas }\end{array}$} \\
\hline \multirow{4}{*}{ SA } & CEV & 121 & 137 & 104 & 122 & 129 & 91 & 82 & 95 & 87 & 80 & 1048 \\
\hline & CNE & 35 & 38 & 40 & 32 & 38 & 28 & 28 & 36 & 41 & 34 & 350 \\
\hline & CMD & 10 & 15 & 8 & 6 & 3 & 3 & 14 & 5 & 2 & 1 & 67 \\
\hline & Total & 166 & 190 & 152 & 160 & 170 & 122 & 124 & 136 & 130 & 115 & 1465 \\
\hline \multirow{4}{*}{ MN } & CEV & 292 & 239 & 235 & 240 & 231 & 234 & 227 & 221 & 211 & 226 & 2356 \\
\hline & CNE & 106 & 101 & 93 & 109 & 85 & 88 & 87 & 81 & 97 & 123 & 970 \\
\hline & CMD & 7 & 15 & 6 & 7 & 2 & 7 & 6 & 11 & 2 & 7 & 70 \\
\hline & Total & 405 & 355 & 334 & 356 & 318 & 329 & 320 & 313 & 310 & 356 & 3396 \\
\hline \multirow{4}{*}{ LI } & CEV & 161 & 152 & 134 & 128 & 113 & 120 & 113 & 129 & 111 & 101 & 1262 \\
\hline & CNE & 50 & 33 & 42 & 40 & 44 & 47 & 35 & 41 & 38 & 36 & 406 \\
\hline & CMD & 8 & 18 & 7 & 3 & 10 & 3 & 9 & 6 & 4 & 3 & 71 \\
\hline & Total & 219 & 203 & 183 & 171 & 167 & 170 & 157 & 176 & 153 & 140 & 1739 \\
\hline \multirow{4}{*}{ CE } & CEV & 139 & 112 & 103 & 115 & 94 & 85 & 101 & 68 & 114 & 91 & 1022 \\
\hline & CNE & 30 & 40 & 47 & 37 & 36 & 39 & 32 & 31 & 46 & 50 & 388 \\
\hline & CMD & 11 & 8 & 12 & 3 & 10 & 8 & 7 & 6 & 8 & 5 & 78 \\
\hline & Total & 180 & 160 & 162 & 155 & 140 & 132 & 140 & 105 & 168 & 146 & 1488 \\
\hline
\end{tabular}

Legenda: MR: Macrorregiões; SA: Semiárido; MN: Meio-norte; LI: Litoral; CE: Cerrados; CEV: Causas evitáveis; CNE: Causas não evitáveis; CMD: Causas mal-definidas.

Fonte: Costa REAR, et al., 2019; dados extraídos do Sistema de Informações sobre Mortalidade (SIM).

\section{DISCUSSÃO}

O presente estudo apontou queda da mortalidade infantil no estado do Piauí em todas as macrorregiões entre 2008 e 2017. Fenômeno semelhante é observado no país como um todo, e é relacionado a diversas melhorias na qualidade de vida e atenção à saúde diretamente relacionadas à mortalidade infantil, como alimentação e nutrição, saneamento básico, vacinação e modelo de atenção à saúde, à mãe e ao recémnascido (SILVA AL e MATHIAS TAF, 2014). Apesar disso, segundo o IBGE, a Taxa de Mortalidade Infantil (TMI) para o estado em 2016, foi de 19,1/1000 nascidos vivos, enquanto a do Brasil, foi de 13,3/1000 nascidos vivos no mesmo período (IBGE, 2020). 
Levando-se em conta que a Organização Mundial da Saúde (OMS) considera inaceitável uma TMI superior a 10/1000 nascidos vivos, nota-se que a mortalidade infantil consiste em um importante problema de saúde pública na realidade brasileira e nacional (UN, 2015). Em todos os anos do período considerado e em todas as macrorregiões do estado, o grupo mortalidade por causas evitáveis foi o grupo de causas que apresentou o maior número de óbitos, assim como o grupo neonatal, considerando-se as faixas etárias. Outros estudos realizados na região Nordeste do Brasil, como em Recife-PE e no estado da Bahia, que apresentam realidade socioeconômica e de acesso à saúde semelhantes ao Piauí, apresentaram resultados semelhantes (MARQUES LJP, et al., 2018; TAVARES LT, et al., 2016).

A mortalidade infantil por causas evitáveis analisa óbitos em menores de 1 ano potencialmente preveníveis com os atuais recursos tecnológicos disponíveis, caso as mães tivessem adequado acesso aos mecanismos de promoção, prevenção e atenção à saúde, assim como a uma melhor qualidade vida (PACHECO CP, 2010; FERRARI RAP e BERTOLOZZI MR, 2012; GUIMARÃES TMR, et al., 2009).

O Índice de Desenvolvimento Humano (IDH) é um indicador que avalia uma série de parâmetros socioeconômicos, como expectativa de vida, renda e escolaridade (PNUD BRASIL, 2019; PNUD BRASIL, 2019; PNUD BRASIL, 2019). O índice de cobertura do Programa em Saúde da Família, por sua vez, é um importante indicador do acesso a serviços essenciais em saúde, pois os serviços disponibilizados por essa estratégia permitem o acompanhamento em saúde e também o encaminhamento para serviços adequados de maneira pública e universal (GUIMARÃES TMR, et al., 2009).

Cabe destacar que o estado do Piauí figura como um dos mais baixos IDHs do Brasil, com 81,3\% dos municípios com Índice de Desenvolvimento Humano Municipal (IDHM) considerados baixo ou muito baixo, o que está associado a mau perfil socioeconômico em grande parte dos seus municípios, apesar de apresentar uma considerável cobertura das famílias pelo PSF desde de sua criação (IBGE, 2010; BRASIL, 2019; PNUD BRASIL, 2019; PNUD BRASIL, 2019; PNUD BRASIL, 2019).

Em regiões em que muitas mães e crianças vivem em más condições ambientais e com dificuldade de acesso a serviços de saúde de qualidade, é esperado um grande número de óbitos infantis por causas evitáveis, como foi ratificado pelos dados levantados nesta pesquisa (IBGE, 2010; PNUD BRASIL, 2019; PNUD BRASIL, 2019; PNUD BRASIL, 2019; BARRETO JOM e NERY IS, 2010; BARRETO JOM, et al., 2012).

Analisando-se as categorias de causas dos óbitos infantis evitáveis (CID-10), notou-se que, apesar das flutuações de cada categoria, houve decréscimo, especialmente nos óbitos reduzíveis por adequada atenção à mulher na gestação e no parto, podendo-se, a partir desse dado, inferir que houve melhoria na atenção à saúde entre 2008 e 2017. Entretanto, a prevalência de óbitos infantis evitáveis em decorrência de má atenção à mulher na gestação ainda é considerada alta, necessitando-se de políticas mais incisivas nessa área, como maior estímulo à realização de consultas no pré-natal e melhoria de sua qualidade.

Por sua vez, a baixa ocorrência de óbitos infantis reduzíveis por ações de imunização no período considerado, ratifica a boa cobertura vacinal, bem como a importância das ações de imunização promovidas pelo Sistema Único de Saúde (SUS) no Piauí na redução da mortalidade infantil por causas evitáveis nos últimos anos (SARDINHA LMV, 2014; GUIMARÃES TMR, et al., 2009; BARRETO JOM, et al., 2012; SOUZA MFM, et al., 2018; BARRETO JOM e NERY IS, 2015; CARETI CM, et al., 2014; SILVA PLN, et al., 2018; FILHO ACAA, et al., 2017; CANABRAVA PBE, et al., 2016; FILHO ACAA, et al., 2018).

Observou-se relação direta entre o percentual de óbitos infantis evitáveis e a porcentagem de munícipios com IDHM baixo e muito baixo, enquanto houve relação inversa entre estes óbitos e número de indivíduos cadastrados no Programa Saúde da Família, evidenciando-se a contribuição de determinantes socioeconômicos na ocorrência de óbitos infantis por causas evitáveis nesse período e uma importância extremamente relevante do acesso à saúde básica, por meio do PSF e seus serviços, para minimizar a mortalidade infantil por causas evitáveis entre 2008 e 2017 (SARDINHA LMV, 2014; PNUD BRASIL, 2019; PNUD BRASIL, 2019; PNUD BRASIL, 2019; BARRETO JOM, et al., 2012; SOUZA MFM, et al., 2018; BARRETO JOM e NERY IS, 2015; CARETI CM, et al., 2014; SILVA PLN, et al., 2018; FILHO ACAA, et al., 2017; CANABRAVA PBE, et al., 2016; FILHO ACAA, et al., 2018 ). 
As mortes infantis são relacionadas a fatores biológicos, socioeconômicos, atenção à saúde, ao pré-natal, ao parto, à criança no primeiro ano de vida, comportamentais da mãe e demográficos. Dessa forma, nota-se que a maior parte dos fatores é passível de intervenção e relacionada ao padrão de vida da população (SARDINHA LMV, 2014; PACHECO CP, 2010; SILVA AL e MATHIAS TAF, 2014).

O IDHM, ao avaliar renda, escolaridade e expectativa de vida, e o número de indivíduos cadastrados no Programa de Saúde da Família (PSF), que cobre uma ampla gama de serviços de saúde básicos, servem como ótimos indicadores da qualidade de vida e de saúde de uma população pela abrangência de determinantes circunscritos (PNUD BRASIL, 2019; PNUD BRASIL, 2019; PNUD BRASIL, 2019; SOUZA MFM, et al., 2018; BARRETO JOM e NERY IS, 2015; CARETI CM, et al., 2014).

Este estudo tem como limitações o viés de subnotificação e/ou inadequado fornecimento dos dados para a base por se tratar de estudo epidemiológico com utilização de fonte de dados secundários (CANABRAVA PBE, et al., 2016). Além disso, também deve-se destacar que existem parâmetros socioeconômicos e de acesso à saúde não circunscritos pelo IDHM e cobertura do PSF (PNUD BRASIL, 2019; PNUD BRASIL, 2019; PNUD BRASIL, 2019; GUIMARÃES TMR, et al., 2009).

Como ponto forte, foram obtidas informações referentes aos óbitos infantis e nascidos vivos em um período de 10 anos, o que permitiu avaliar a mortalidade infantil de todo o estado do ponto de vista histórico, assim como foi possível correlacioná-la linearmente com indicadores socioeconômicos e assistenciais em saúde que abrangem um grande número de determinantes.

\section{CONCLUSÃO}

Apesar de ter havido redução percentual no número de óbitos infantis no Piauí e em cada uma de suas 4 macrorregiões entre 2008 e 2017, a mortalidade infantil neste estado ainda foi elevada e amplamente constituída por óbitos infantis neonatais e por causas evitáveis. Sexo masculino e cor/raça preta ou parda foram aqueles com maior número de óbitos infantis no estado do Piauí e suas macrorregiões. Neste estudo, evidenciou-se que quanto maior a porcentagem de municípios com IDHM muito baixo e baixo e menor a cobertura do PSF em uma macrorregião piauiense, maior foi o percentual de óbitos infantis por causas evitáveis, relações diretas provadas pelo coeficiente de correlação linear de Pearson, o que demonstrou a importância de determinantes socioeconômicos e de saúde na mortalidade infantil por causas evitáveis. Levando-se em conta os números absolutos e relativos da mortalidade infantil por causas evitáveis no estado do Piauí e suas macrorregiões entre 2008 e 2017 e as correlações realizadas neste estudo, pode-se inferir que são necessários, neste estado, melhorias socioeconômicas e dos serviços de saúde.

\section{AGRADECIMENTOS E FINANCIAMENTO}

Este projeto foi financiado com recursos do Programa Institucional de Bolsas de Iniciação Científica (PIBIC) da Universidade Estadual do Piauí (UESPI) [Edital PROP № 001/2019]. Dessa forma, agradecemos à instituição pelo apoio financeiro por meio da bolsa de iniciação científica do programa na modalidade PIBICUESPI.

\section{REFERÊNCIAS}

1. BARRETO JOM, et al. Estratégia de saúde da família e internações hospitalares em menores de 5 anos no Piauí, Brasil. Cad. Saúde Pública, 2012; 28(3): 515-526.

2. BARRETO JOM, NERY IS. Mortalidade infantil no Piauí: reflexo das desigualdades sociais e iniquidades em saúde. In: Lima SO, Ferreira MDM. Direitos humanos em políticas públicas. 1a ed. Teresina: Editora Universidade Federal do Piauí, 2010; 19 p.

3. BARRETO JOM, NERY IS. Óbitos infantis em um estado do Nordeste brasileiro: características e evitabilidade. Tempus, Actas de saúde colet, 2015; 9(3): 9-19.

4. BRASIL. 2019. In: Departamento de Informática do Sistema Único de Saúde (Estatísticas vitais). Brasília: Ministério da Saúde. 
5. CANABRAVA PBE, et al. Mortalidade infantil por causas evitáveis no Distrito Federal no período de 2003 a 2012 . Rev Med Saude. Brasilia, 2016; 5(2): 192-202.

6. CARETI CM, et al. Perfil da mortalidade infantil a partir da investigação de óbitos. Rev. Eletr. Enf, 2014; 16(2): 352360.

7. CARVALHO RAS, et al. Desigualdades em saúde: condições de vida e mortalidade infantil em região do nordeste do Brasil. Rev. Saúde Pública, 2015; 49(1): 1-9.

8. COSTA REAR, et al. Evolução epidemiológica da COVID-19 no Estado do Piauí em 2 meses. Research, Society and Development, 2020; 9(8): 1-13.

9. FERRARI RAP, BERTOLOZZI MR. Mortalidade pós-neonatal no território brasileiro: uma revisão da literatura. Rev. esc. enferm, 2012; 46(5): 1207-1214.

10. FILHO ACAA, et al. Aspectos epidemiológicos da mortalidade infantil em um estado do Nordeste do Brasil. Enfermería Global, 2018; 49(1): 458-467.

11. FILHO ACAA, et al. Mortalidade Infantil em uma capital do Nordeste Brasileiro. Enferm. Foco, 2017; 8(1): 32-36.

12. FILHO DBF, JÚNIOR JAS. Desvendando os mistérios do Coeficiente de Correlação de Pearson (r). Revista Política Hoje, 2009; 18(1): 115-146.

13. GUIMARÃES TMR, et al. Impacto das ações de imunização pelo programa Saúde da Família na mortalidade infantil por doenças evitáveis em Olinda, Pernambuco, Brasil. Cad. Saúde Pública, 2009; 25(4): 868-876.

14. INSTITUTO BRASILEIRO DE GEOGRAFIA E ESTATÍSTICA (IBGE). 2010. In: Censo demográfico 2010: Piauí. Rio de Janeiro: Instituto Brasileiro de Geografia e Estatística.

15. INSTITUTO BRASILEIRO DE GEOGRAFIA E ESTATÍSTICA (IBGE). 2020. In: Cidades e Estados: Piauí. Rio de Janeiro: Instituto Brasileiro de Geografia e Estatística.

16. MARQUES LJP, et al. Concordância da causa básica e da evitabilidade dos óbitos infantis antes e após a investigação no Recife, Pernambuco, 2014. Epidemiol. Serv. Saúde, 2018; 27(1):1-8.

17. NASCIMENTO SG, et al. Mortalidade infantil por causas evitáveis em uma cidade do Nordeste do Brasil. Rev. Bras. Enferm, 2014; 67(2): 208-212.

18. PACHECO CP. Evolução da mortalidade infantil, segundo óbitos evitáveis: Macrorregiões de Saúde do Estado de Santa Catarina, 1997 - 2008. Dissertação (Mestrado em Saúde Pública) - Faculdade de Saúde Pública. Universidade de São Paulo, São Paulo, 2010; 123 p.

19. PNUD BRASIL. 2019. In: Desenvolvimento Humano nas macrorregiões. Brasília: PNUD Brasil.

20. PNUD BRASIL. 2019. In: O que é o IDH. Brasília: PNUD Brasil.

21. PNUD BRASIL. 2019. In: O que é o IDHM. Brasília: PNUD Brasil.

22. REDE INTERAGENCIAL DE INFORMAÇÃO PARA A SAÚDE (RIPSA). 2008. In: Indicadores básicos para a saúde no Brasil: conceitos e aplicações. Brasília: Organização Pan-Americana da Saúde.

23. RUOFF AB, et al. O processo de análise da evitabilidade dos casos de óbito infantil e fetal: estudo de caso único. Texto Contexto Enferm, 2018; 27(4): 1-8

24. SANDERS LSC, et al. Mortalidade infantil: análise de fatores associados em uma capital do Nordeste brasileiro. Cad. Saúde Colet, 2017; 25(1): 83-89.

25. SARDINHA LMV. Mortalidade infantil e fatores associados à atenção à saúde: estudo caso-controle no Distrito Federal (2007-2010). Tese (Doutorado em Medicina Tropical) - Faculdade de Medicina. Universidade de Brasília, Brasília, 2014; 181 p.

26. SILVA AL, MATHIAS TAF. Fatores de risco independentes associados a óbitos infantis. Acta Paul Enferm, 2014; 27(1): 48-55.

27. SILVA PLN, et al. Evitabilidade da mortalidade infantil na região de saúde de Janaúba/Monte Azul, Minas Gerais, Brazil. J. Health Biol Sci, 2018; 6(1): 35-41.

28. SOUZA MFM, et al. Transição da saúde e da doença no Brasil e nas Unidades Federadas durante os 30 anos do Sistema Único de Saúde. Ciência \& Saúde Coletiva, 2018; 23(6): 1737-1750.

29. SZWARCWALD CL, et al. Mortalidade infantil no Brasil: Belíndia ou Bulgária? Cad Saúde Públ, 1997; $13(3): 503-516$.

30. TAVARES LT, et al. Mortalidade infantil por causas evitáveis na Bahia, 2000-2012. Rev Eletron Comun Inf Inov Saúde, 2016; 10(3): 1-10.

31. UNITED NATIONS (UN). 2015. In: The Millennium Development Goals Report 2015. New York: UNITED NATIONS. 